\title{
Modulation of Inflammatory Responses by Wnt/ $\beta$-Catenin Signaling in Dendritic Cells: A Novel Immunotherapy Target for Autoimmunity and Cancer
}

\author{
Amol Suryawanshi ${ }^{1+}$, Raghu K. Tadagavadi ${ }^{2}$, Daniel Swafford ${ }^{1}$ and \\ Santhakumar Manicassamy ${ }^{1 *}$
}

OPEN ACCESS

Edited by: Victor Manuel Baizabal-Aguirre, Universidad Michoacana de San Nicolás de Hidalgo, Mexico

Reviewed by:

John P. Vasilakos, 3M Drug Delivery Systems

Division, USA

Amanda Jane Gibson, Royal Veterinary College, UK

Tracy L. McGaha,

University of Toronto, Canada

*Correspondence:

Santhakumar Manicassamy smanicassamy@gru.edu

tPresent address: Amol Suryawanshi, Foundational Immunology, AbbVie Inc., Worcester, MA, USA

Specialty section:

This article was submitted to Molecular Innate Immunity, a section of the journal

Frontiers in Immunology

Received: 01 August 2016 Accepted: 12 October 2016 Published: 27 October 2016

Citation:

Suryawanshi A, Tadagavadi RK, Swafford D and Manicassamy S (2016) Modulation of Inflammatory Responses by Wnt/ $\beta$-Catenin Signaling in Dendritic Cells: A Novel Immunotherapy Target for Autoimmunity and Cancer.

Front. Immunol. 7:460 doi: 10.3389/fimmu.2016.00460
'Cancer Immunology, Inflammation, and Tolerance Program, Georgia Cancer Center, Augusta University, Augusta, GA, USA, ${ }^{2}$ Janssen Research \& Development, Spring House, PA, USA

The $\mathrm{Wnt} / \beta$-catenin pathway is an evolutionarily conserved signaling pathway critical for several biological processes. An aberrant Wnt/ $\beta$-catenin signaling is linked to several human diseases. Emerging studies have highlighted the regulatory role of the Wnt/ $\beta$-catenin signaling pathway in normal physiological processes of parenchymal and hematopoietic cells. Recent studies have shown that the activation of $\mathrm{Wnt} / \beta$-catenin pathway in dendritic cells (DCs) play a critical role in mucosal tolerance and suppression of chronic autoimmune pathologies. Alternatively, tumors activate $\mathrm{Wnt} / \beta$-catenin pathway in DCs to induce immune tolerance and thereby evade antitumor immunity through suppression of effector $T$ cell responses and promotion of regulatory $T$ cell responses. Here, we review our work and current understanding of how Wnt/ $\beta$-catenin signaling in DCs shapes the immune response in cancer and autoimmunity and discuss how Wnt/ $\beta$-catenin pathway can be targeted for successful therapeutic interventions in various human diseases.

Keywords: immunotherapy, Wnt, $\beta$-catenin, dendritic cells, Immune-tolerance, Immuno-oncology, autoimmune diseases, antitumor immunity

\section{INTRODUCTION}

The immune system functions constantly to defend from pathogens while preserving tolerance to self-antigens and commensal microbes $(1,2)$. Activation of the immune system in response to pathogens or sterile inflammation involves a cascade of innate and adaptive immune responses that are precisely controlled through a complex network of regulatory mechanisms $(3,4)$. Aberrations in immune-regulatory pathways result in different conditions including cancer and autoimmune diseases. The immune system is equipped with diverse cell types and secreted factors to achieve constant surveillance against pathogens while maintaining tolerance to self. Proinflammatory leukocyte subsets, such as monocytes, NK cells, and cytotoxic T cells, mainly provide protection from pathogens and cancer, whereas various subsets of regulatory $\mathrm{T}$ cells check untoward inflammatory mechanisms. Unlike other leukocytes, dendritic cells (DCs) possess the ability to elicit both proand anti-inflammatory functions made possible by the presence of various regulatory mechanisms, including crosstalk between Toll-like receptor (TLR)- and Wnt/ $\beta$-catenin signaling pathways. 
Dendritic cells are crucial in the induction of immunity and tolerance. Every tissue in humans is armed with DCs to sense and induce inflammatory immune responses against pathogens or to preserve a tolerogenic environment under steady state conditions. DCs equipped with pattern-recognition receptors (PRRs) play a central role in detection of pathogen-associated molecular patterns (PAMPs) and induction of robust host immunity through a controlled activation of different arms of the innate and adaptive immune responses $(5,6)$. DCs as professional antigen-presenting cells (APCs) recognize PAMPs through membrane-bound TLRs and C-type lectin receptors (CLRs) or cytoplasmic NOD-like receptors (NLRs) and RIG-1-like receptors (RLRs) (2, 3, 4).

Recognition of pathogens by DCs leads to phagocytosis of microbes, cytokine production, and expression of antigen presentation and costimulatory molecules, ultimately causing selective polarization of adaptive immune responses based on the type of pathogen. Accordingly, intracellular infections promote IFN- $\gamma$ and IL-12 production by DCs that activates IFN- $\gamma$-producing $\mathrm{CD}^{+} \mathrm{T}$ helper 1 (Th1) and $\mathrm{CD}^{+}$cytotoxic $\mathrm{T}$ cell responses, whereas extracellular pathogens activate DCs to induce IL-4-producing $\mathrm{CD}^{+}{ }^{+} \mathrm{T}$ helper 2 (Th2) cell responses $(4,7)$. Alternatively, DCs can induce IL- $17 \mathrm{~A}^{+} \mathrm{CD} 4^{+} \mathrm{T}$ helper 17 (Th17) cell responses against fungi and extracellular bacteria through increased production of IL- 6 , TGF- $\beta$, and IL-23 $(3,4,8)$.

In addition to induction of immunity against pathogens, DCs contain effector immune responses and maintain peripheral tolerance to self-antigens, commensal microorganisms, and dietary components $(2,6)$. DCs generate tolerance and control chronic inflammatory responses by various mechanisms, including induction of anergic or regulatory $\mathrm{T}$ cell responses, and regulating effector Th1, Th2, and Th17 cell responses through differential expression of costimulatory molecules and secretion of regulatory cytokines $(2,6)$. Although significant progress has been achieved in our understanding on DCs' diverse mechanisms in eliciting effector T cell responses in immunity, the signaling networks that program DCs into a regulatory state are poorly defined. In this regard, recent studies have discovered the critical functions of the Wnt/ $\beta$-catenin signaling pathway of DCs in regulation of immune responses in various pathological conditions. Here, we review our current understanding and significance of Wnt/ $\beta$-catenin signaling in DCs in the induction of immune tolerance in physiological conditions, autoimmuninty, and oncogenesis.

\section{Wnt/p-CATENIN SIGNALING}

Wnt $/ \beta$-catenin signaling plays a critical role in cell differentiation, growth, proliferation, survival, and immune cell function $(9,10)$. Wnts are secreted lipid-modified glycoproteins that bind to seven-pass transmembrane Frizzled (Fzd) family receptors and activate multiple signaling pathways. In humans, there are $19 \mathrm{Wnt}$ proteins and 10 cognate Fzd receptors $(10,11)$. The binding of Wnts to Fzd receptors activates canonical and/or non-canonical signaling pathways (Figure 1) $(9,10,12)$. Low-density lipoprotein receptor-related protein 5 (LRP5) and LRP6 co-receptors are essential for optimal activation of canonical Wnt/ $\beta$-catenin signaling $(10,13,14)$. Wnt interaction with Fzd receptors results in cytoplasmic accumulation of $\beta$-catenin and its translocation into the nucleus, where it interacts with T-cell factor/lymphoid enhancer factor (TCF/LEF) and regulates the transcription of several target genes $(9,12)$. Wnt ligands also can stimulate other non-canonical signaling pathways independent of $\beta$-catenin activation (11).

In the absence of Wnt and Fzd interaction, $\beta$-catenin actively synthesized in the cytoplasm is removed through a destruction complex $(9,10,14)$. This destruction complex consists of scaffolding proteins, such as axin and adenomatous polyposis coli (APC), and Ser/Thr kinases such as casein kinase $1 \alpha(\mathrm{CK} 1 \alpha)$ and glycogen synthase kinase $3 \beta$ (GSK3 $\beta)(9,10,13)$. GSK-3 $\beta$ phosphorylates $\beta$-catenin, leading to ubiquitination and proteasomal degradation mediated by $\beta$-transducin repeat-containing $(\beta-\operatorname{TrCP})$ protein and Wilms tumor suppressor protein complex (Wtx) (9-11). Wnt binding of the Fzd receptor leads to recruitment of co-receptors LRP5/6 and is followed by recruitment of disheveled (DVL) to the cytoplasmic tail of LRP5/6 $(9,10,11,13)$. These signaling events inactivate the destruction complex by recruiting it to the plasma membrane. Moreover, GSK3 $\beta$ in the destruction complex phosphorylates LRP5/6 instead of $\beta$-catenin, leading to increased accumulation of $\beta$-catenin in the cytoplasm $(9,10,11,13)$.

Thus, Wnts activate $\beta$-catenin signaling through inhibition of $\beta$-catenin destruction, resulting in its cytoplasmic accumulation and translocation to the nucleus. In the nucleus, $\beta$-catenin displaces the co-repressor molecule Groucho from the TCF/LEF complex and activates transcription of target genes $(9,10,11,13)$.

\section{Wnt-INDEPENDENT $\beta$-CATENIN SIGNALING IN DCs}

\section{E-Cadherin in $\beta$-Catenin Activation}

In addition to Wnt-mediated activation of $\beta$-catenin, there are numerous pathways that function independent of Wnt ligands involved in activation of $\beta$-catenin in DCs (Figure 2). For example, disruption of E-cadherin-homophilic interactions in DCs activates $\beta$-catenin, which in turn programs DCs to a regulatory state (15). Importantly, these regulatory DCs produce high levels of IL-10 in response to LPS and protect mice from experimental autoimmune encephalomyelitis (EAE) (15). The cytoplasmic domain of E-cadherin is known to interact with and sequester $\beta$-catenin (16). This would therefore limit $\beta$-catenin signaling in a manner independent of Wnt ligands or axin destruction complex expression. E-cadherin-mediated disruption of $\beta$-catenin signaling is further supported by observations of phosphorylation and degradation of $\beta$-catenin by destruction complexes localized primarily at adherens junctions (17). This suggests a significant link between E-cadherin interactions and canonical Wnt signaling.

Additional binding factors also may mediate this function of E-cadherins at adherens junctions. Caveolin-1, for example, may be recruited by E-cadherin in order to support inhibition of $\beta$-catenin signaling (18). In a recent study, casein kinase 1 (CK1) was shown to phosphorylate another E-cadherin-associated protein known as p120-catenin, which in response to Wnt3a binding stabilizes E-cadherin interactions (19). This subsequently aids in recruitment of CK1 to the LRP5/6 co-receptor for canonical Wnt signaling pathway activation (19). In addition, 


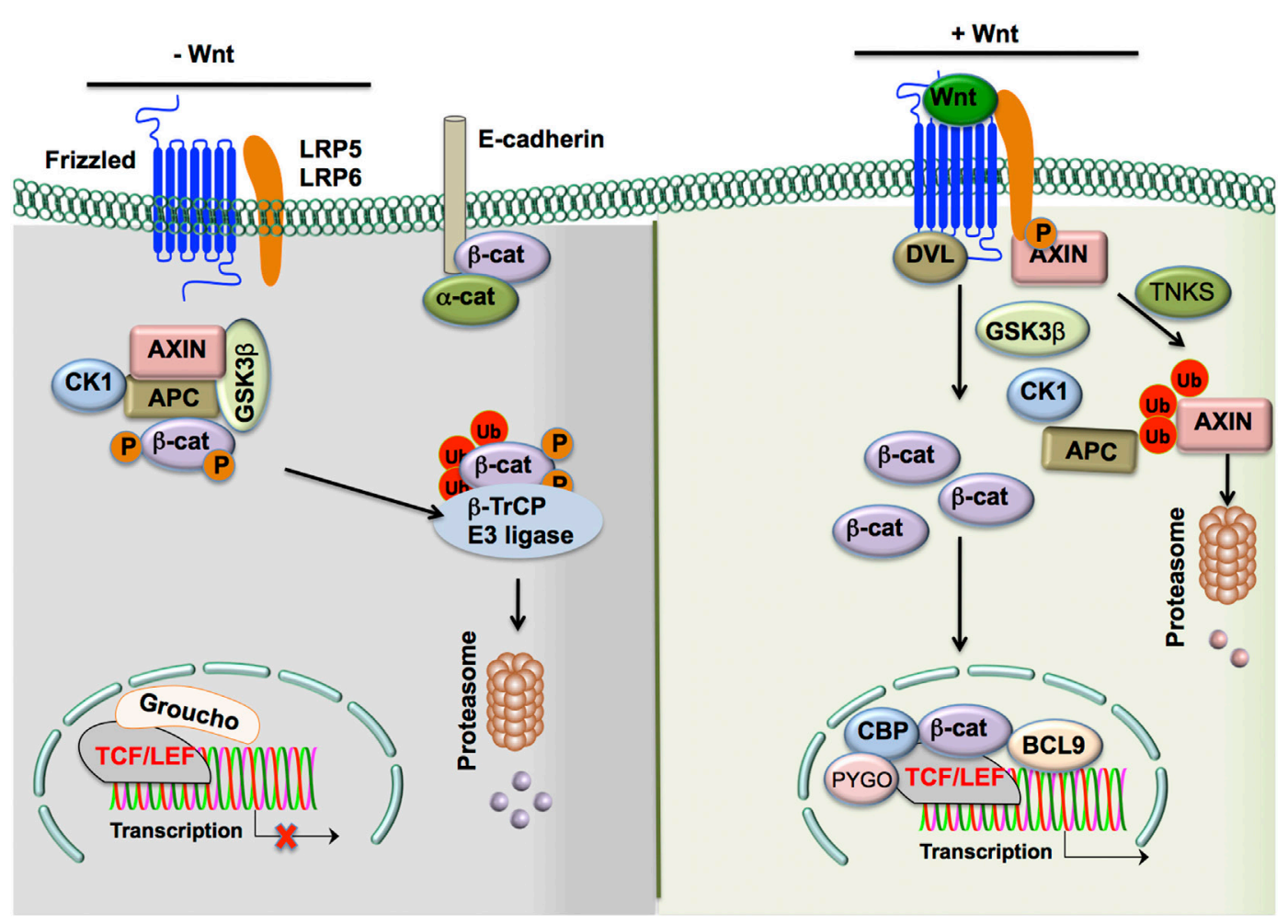

FIGURE 1 | The Wnt/ק-catenin signaling pathway. In the absence of Wnt ligands, $\beta$-catenin levels in the cytoplasm are largely reduced by the following means: (1) a destruction complex consisting of the scaffolding proteins APC and AXIN, as well as Ser/Thr kinases CK1 $\alpha$ and GSK3 $\beta$, interacts with $\beta$-catenin in the cytosol to induce phospho-inactivation of the protein by the two Ser/Thr kinases. (2 and 3) Phospho- $\beta$-catenin is promoted to ubiquitination and subsequent proteasomal degradation by $\beta$-Trcp and WTX proteins. In contrast, in the presence of Wnt ligands, $\beta$-catenin levels in the cytoplasm are upregulated and actively participate in cell signaling through the following steps: (1) Wnts bind to cognate Fzd receptors and interact with adjacent LRP5/6 co-receptors, (2) the resulting complex recruits DVL to the cytoplasmic tail of Fzd receptors, and (3) DVL recruits the destruction complex to the Fzd/LRP5/6 complex and associated Ser/Thr kinases such as GSK3 $\beta$ phosphorylate the co-receptor instead of $\beta$-catenin due to the altered localization of the complex. Moreover, cytoplasmic TNKS ubiquitinates Axin, targeting it for proteasomal degradation and causing disruption of the $\beta$-catenin destruction complex. (4) Cytosolic $\beta$-catenin levels are free to accumulate and subsequently translocate to the nucleus. (5) This translocation results in the displacement of the co-repressor Groucho on the TCF/LEF transcription factor by $\beta$-catenin and the recruitment of co-activators such as BCL9, CBP, and PYGO to result in the transcription of target genes. Alternate means of activation of $\beta$-catenin signaling include the disruption of epithelial cadherin (E-cadherin)-E-cadherin interactions, which frees the association of $\beta$-catenin to the cytoplasmic domain of E-cadherin and allows for its accumulation in the cytoplasm. In addition, TLRs and other such transmembrane receptors may result in PI3K/Akt signaling. This leads to an alternative phospho-activation of $\beta$-catenin through phosphorylation at Ser552 and phospho-inactivation of GSK3 $\beta$ at Ser9 to prevent the degradation of $\beta$-catenin by the destruction complex and allow it to accumulate in the cytoplasm and participate in cell signaling. Abbreviations: APC, adenomatous polyposis coli; CK1 $\alpha$, casein kinase $1 \alpha$; GSK3 $\beta$, glycogen synthase kinase $3 \beta$; WTX, Wilms tumor suppressor protein complex; $\beta$-Trcp, $\beta$-transducin repeat-containing protein; TNKS,

tankyrase-1; Fzd, Frizzled; LRP5/6, low-density lipoprotein receptor-related protein 5/6; DVL, disheveled; TCF/LEF, T-cell factor/lymphoid enhancer factor; BCL9, B-cell CLL/ymphoma 9 protein; CBP, CREB-binding protein; PYGO, Pygopus; TLR, Toll-like receptor; PI3K, phosphoinositide-3-kinase.

CK1 phosphorylation of E-cadherin weakens its affinity for $\beta$-catenin, thus increasing free $\beta$-catenin levels in the cytosol for signaling. Elimination of p120-catenin inhibited Wnt-mediated $\beta$-catenin signaling. Unligated E-cadherin may therefore support $\beta$-catenin signaling by helping canonical Wnt signaling in cells. This indicates that the extent of cell-cell interactions mediated by E-cadherins does play a significant role in immunity and tolerance.

\section{TLRs in $\beta$-Catenin Activation}

Activation of TLRs is known to modulate $\beta$-catenin signaling in different immune and non-immune cells (20). In this regard, a recent study has shown that TLR2 signaling through the PI3K/Akt pathway activates $\beta$-catenin in DCs and induces the expression of vitamin A-metabolizing enzymes and IL-10 (Figure 2) (21). Interestingly, activation of TLR2 in DCs promotes T regulatory cell responses and protects mice from Th1/Th17-mediated autoimmune neuroinflammation $(21,22)$. Stimulation of TLR2 present on DCs increased IL-10 production through increased expression and phosphorylation of extracellular signal-regulated kinase (ERK) and mitogen-associated protein kinase (MAPK) $(23,24)$. Phosphorylation of $\beta$-catenin at Ser 552 by Akt activates its nuclear transcription activity, whereas Akt phosphorylation of GSK3 $\beta$ at Ser9 prevents it from marking $\beta$-catenin for degradation $(25,26,27)$. Inhibition of the Akt or Erk pathway individually in DCs showed that $\beta$-catenin signaling via TLR2 stimulation is primarily dependent on Akt but not Erk (21). This shows that $\beta$-catenin signaling works synergistically with other pathways, 


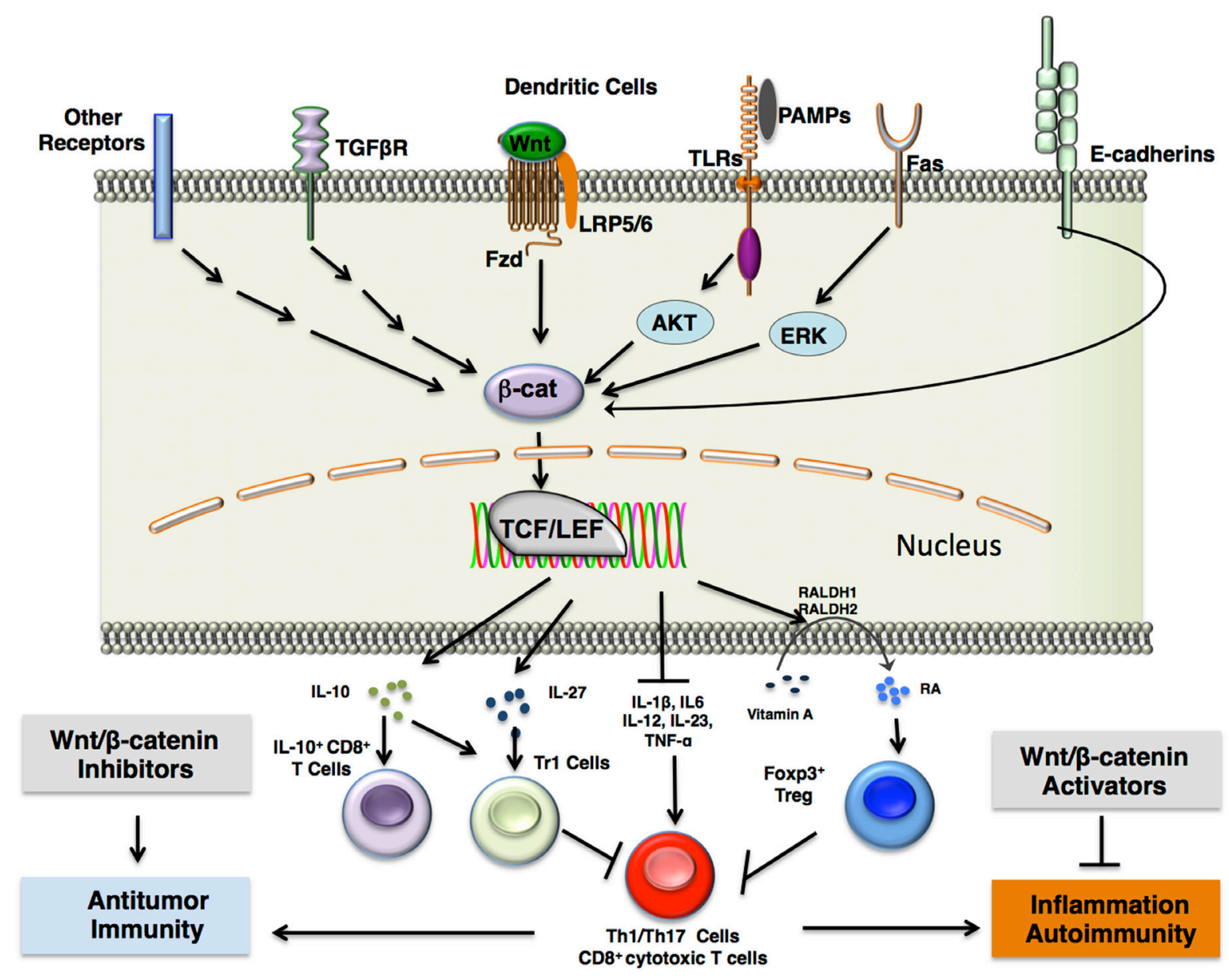

FIGURE 2 | $\boldsymbol{\beta}$-catenin signaling in DCs induces immune tolerance. $\beta$-catenin upregulation is induced in dendritic cells via a number of different receptor interactions, including canonical Wnt/Fzd signaling, stimulation of TLRs such as TLR2 with antigen and subsequent ERK signaling, Fas receptor activation, release of $\beta$-catenin from E-cadherins upon disruption of E-cadherin interactions, or activation of other pattern-recognition receptors such as Fc $\gamma$ receptors or dectin-1. This results in $\beta$-catenin-mediated association of the TCF/LEF transcription factor with various co-activators that lead to transcription of target genes. This further results in the expression of anti-inflammatory cytokines, such as IL-10 and IL-27, which induce type 1 regulatory T (Tr 1 ) cells or CD8+IL-10+ T cells, and TGF- $\beta$, which induces FoxP3 ${ }^{+}$regulatory $T$ (Treg) cells. In addition, $\beta$-catenin signaling in DCs upregulate vitamin A-metabolizing enzymes (RALDH 1/2), which convert vitamin A to retinoic acid (RA). RA induces Treg differentiation. Accumulation of Tregs in the tissue microenvironment may result in inhibition of proinflammatory Th1/Th17 cells or $\mathrm{CD}^{+}$cytotoxic $T$ cells. Pharmacological inhibition of $\beta$-catenin signaling components may thus lead to downregulation of regulatory $T$ cells to mount a more robust effector T cell response against chronic infections and tumors, which may utilize $\beta$-catenin signaling components to engage in immune surveillance evasion. Such inhibitors include TNKS inhibitors, XAV939 and JW55, and PORCN inhibitor, C59. In contrast, pharmacological activation of $\beta$-catenin signaling components may result in upregulation of regulatory T cells to dampen chronic inflammation and autoimmunity. Such activators include the Axin/ $\beta$-catenin interaction disruptor SKL2001 and administration of canonical Wnts. Abbreviations: Fzd, Frizzled; TLR, Toll-like receptor; ERK, extracellular signal-related kinase; Muc, mucin; Gal3, galectin 3; TCF/LEF, T cell factor/lymphoid enhancer factor; IL-10, interleukin 10; IL-27, interleukin 27; FoxP3, forkhead box P3; TGF $\beta$, transforming growth factor $\beta$; RALDH 1/2, retinaldehyde dehydrogenase 1/2; Th1/17 cells, T helper 1/17 cells; TNKS, tankyrase-1; PORCN, porcupine.

such as the Erk pathway, to induce anti-inflammatory cytokines and proliferation of $\mathrm{T}$ regulatory cells. Like TLR2, other TLRs such as TLR3, TLR5, and TLR9 are known to activate or regulate $\beta$-catenin signaling via a mechanism dependent on PI3K/AKT and Erk pathways $(20,21,28)$.

In addition to E-cadherin and TLR stimulation, other pathways such as FAS (29), TGF- $\beta$ (30), and PLC- $\gamma 2$ (31) activate or regulate $\beta$-catenin signaling in DCs. Further studies are warranted to elucidate how these pathways may work in tandem to effectively establish a tolerogenic phenotype in DCs.

\section{Wnt/ $\beta$-CATENIN SIGNALING OF DCs IN THE REGULATION OF TOLERANCE AND INFLAMMATION}

Although activation of effective innate and adaptive immune responses is critical for protective immunity, uncontrolled activation of these immune responses results in chronic autoimmune and inflammatory conditions. Moreover, induction of adaptive immunity against commensal microorganisms at mucosal sites, self-antigens, and food constituents is detrimental to human health 
$(1,2,6)$. In this context, DCs play an important role in inducing peripheral immune tolerance at mucosal surfaces through several regulatory mechanisms $(1,2,4,6)$. Furthermore, DCs contribute to the resolution of inflammation through inhibition of ongoing effector $\mathrm{T}$ cell responses and induction of regulatory $\mathrm{T}$ cell responses. Although past studies have characterized the role of $\mathrm{Wnt} / \beta$-catenin signaling in hematopoiesis, cell differentiation, proliferation, and homeostasis, the role of this pathway in DCs in regulating immune responses is poorly understood. Recently, several studies from others and our lab have begun to explore the function of this regulatory pathway in inducing immune tolerance and its significance during cancer, autoimmunity, and infectious diseases (Figure 2). In the next few sections, we will review recent developments in our understanding of the role of $\mathrm{Wnt} / \beta$-catenin signaling in regulating immune responses in pathological conditions.

\section{Wnt/ $\beta$-CATENIN SIGNALING IN DCs REGULATES MUCOSAL TOLERANCE}

Mucosal tolerance to commensals and food antigens is required for optimum health. Mucosal DCs play an important role in inducing peripheral tolerance. Gut DCs are in constant contact with microbial and food antigens and exhibit impaired immunestimulatory capacity $(2,4,6)$. Wnt/ $\beta$-catenin signaling is essential for intestinal homeostasis including epithelial cell proliferation and maintenance of stem cells (32-36). Studies have shown that aberrant activation of the Wnt/ $\beta$-catenin pathway in intestinal epithelial cells results in uncontrolled proliferation, polyps formation, and the development of colorectal cancer $(33,35,37,38,39)$. Under homeostasis, the intestine expresses several components of the Wnt/ $\beta$-catenin signaling pathway, including different Wnt ligands, Fzd receptors, and LRP5/6 co-receptors (40).

Although several studies have shown the importance of Wnt/ $\beta$-catenin signaling in the maintenance of intestinal epithelial cell homeostasis, the role of this pathway in inducing immune tolerance is poorly defined. In this regard, a recent study showed that $\beta$-catenin is constitutively active in intestinal DCs and macrophages compared to splenic DCs (41). This study demonstrated that expression of $\beta$-catenin in intestinal DCs is critical for the induction of regulatory $\mathrm{T}$ cell responses and suppression of Th1 and Th17 cell responses. Thus, DC-specific deletion of $\beta$-catenin results in increased Th1 and Th17 cells over T regulatory cells in the intestine but not in the spleen (41). Interestingly, the absence of $\beta$-catenin in DCs shifts the balance from anti-inflammatory cytokine production to increased production of proinflammatory cytokines in the intestine, suggesting a tolerogenic role of $\beta$-catenin signaling in gut DCs (41). Moreover, a recent study showed that MUC2, a gel-forming mucin secreted by intestinal goblet cells, mediates mucosal tolerance in the intestine through activation of $\beta$-catenin signaling in DCs (42). This study showed that MUC2 is readily taken in by small intestinal DCs through interaction with Galectin-3, dectin-1, and Fc $\gamma$ RIIB. This activates $\beta$-catenin in DCs to induce mucosal tolerance against intestinal commensal microorganisms. Thus, MUC2-mediated activation of $\beta$-catenin in small intestinal DCs suppressed inflammatory immune responses through suppression of the NF- $\kappa \mathrm{B}$ pathway (42). These studies indicate the vital role of $\beta$-catenin signaling not only in epithelial cell homeostasis but also in the maintenance of gut tolerance through regulatory effects of DCs. Further studies on the role of commensal-derived factors, Wnt ligands, mucosal proteins, and various dietary ligands on activation of this pathway will identify novel regulatory mechanisms by which a normal intestine maintains tolerance. These studies will also help in designing better DC-based immunotherapy treatment approaches for enteric pathogens as well as chronic inflammatory conditions of the gut.

\section{Wnt/ק-CATENIN SIGNALING IN DCs: A POTENTIAL THERAPEUTIC TARGET FOR CHRONIC INFLAMMATION}

Although mucosal tolerance is actively enforced all the time to achieve optimum health, dysregulation of the host immune system and commensal microflora results in the development of inflammatory bowel diseases (IBDs) such as ulcerative colitis and Crohn's disease (43). In IBD, intestinal DCs and macrophages lose their tolerogenic properties, resulting in uncontrolled intestinal inflammation (43). Accordingly, our recent study showed that mice lacking $\beta$-catenin in DCs are more susceptible to dextran sodium sulfate (DSS)-induced colitis, a mouse model for IBD, compared to $\beta$-catenin-sufficient mice, indicating the critical role of this pathway in regulation of inflammatory diseases (41). DC-specific deletion of $\beta$-catenin in mice led to increased body weight loss in response to DSS treatment with severe colonic epithelial layer destruction and increased Th 1 and Th 17 cell responses (41). Although this study identified the immune-regulatory role of $\beta$-catenin in DCs in inducing mucosal tolerance and protection against IBD, further studies are warranted to determine upstream and downstream signaling components for $\beta$-catenin activation in intestinal DCs.

Multiple sclerosis (MS) is a chronic autoimmune neuroinflammatory disease that leads to multifocal demyelination in the white matter of the human CNS with debilitating motor and sensory dysfunction $(44,45)$. Previous studies using EAE, a mouse model for MS, have shown that DCs play a critical role in initiation and development of CNS pathology through activation of effector $\mathrm{T}$ cells in the draining lymph nodes (DLN) and CNS $(44,45)$. These studies have shown that DCs contribute to CNS pathology through differentiation and activation of naive $\mathrm{CD} 4^{+} \mathrm{T}$ cells to myelin-specific effector Th1 and Th17 cells $(44,46)$. Furthermore, studies have also indicated the critical role of $\mathrm{CD}^{+} \mathrm{T}$ cells in causing EAE and MS pathogenesis $(47,48)$. Although the role of DCs in initiating effector T cell responses during EAE and MS is well characterized, emerging evidence indicates that DCs are also critical in resolving inflammation and in limiting immunemediated pathology in EAE. DCs play a regulatory role through production of immune-regulatory factors, T regulatory cell activation, and fine-tuning of effector Th1 and Th17 cell responses $(2,4,6)$. However, the molecular signaling networks and counterregulatory mechanisms that induce these regulatory responses are poorly defined. Particularly, the immune modulatory factors 
in the local DLN and tissue microenvironment that are critical in programing DCs to induce tolerance during EAE and MS remain to be understood.

Recent studies from our laboratory have identified the critical function of $\mathrm{Wnt} / \beta$-catenin signaling and the role of TLR2-mediated activation of $\beta$-catenin in regulating ongoing effector immune responses and CNS pathology $(21,49)$. Our data suggest that excessive neuroinflammation is controlled through increased Wnt ligand expression as a feedback mechanism to counter ongoing inflammatory responses. Thus, Wnt3a and Wnt5a expression is significantly upregulated in the DLN during the EAE induction phase and in the CNS during the effector phase. Interestingly, previous studies have shown that the dysregulated Wnt signaling leads to neurodegenerative and inflammatory disorders (50). Furthermore, it has been reported that sustained activation of the Wnt pathway restrains inflammation, stimulates neuroprotection, and promotes neurogeneration (49, 50 ). However, the role of $\mathrm{Wnt} / \beta$-catenin in specific cell types has not been identified during ongoing EAE pathology. Results from our study show that DC-specific deletion of LRP5/6 or $\beta$-catenin leads to early onset as well as increased EAE pathology, indicating the critical role of canonical Wnt-mediated $\beta$-catenin activation in DCs in limiting CNS pathology $(21,49)$. Mechanistically, the absence of Wnt/ $\beta$-catenin signaling in DCs lead to an increased proinflammatory cytokine production in the DLN and CNS, as well as increased effector Th1, Th17, and CD8 ${ }^{+} \mathrm{T}$ cell responses and diminished production of anti-inflammatory cytokines such as IL-10, TGF- $\beta$, and IL-27 (49). Furthermore, transgenic mice with a constitutively active form of $\beta$-catenin in DCs showed diminished neuroinflammation with reduced effector and increased regulatory $\mathrm{T}$ cell responses confirming the regulatory role of $\beta$-catenin signaling in DCs. Moreover, prophylactic and therapeutic treatment with $\beta$-catenin agonists in EAE-induced mice showed diminished EAE pathology, suggesting that immunotherapies targeting Wnt/ $\beta$-catenin signaling specifically in DCs could represent a promising therapeutic approach for clinical management of MS.

Interestingly, Wnt5a, which signals independently of the $\beta$-catenin pathway, also reprograms DCs to limit the expression of inflammatory cytokines $(51,52)$. It is possible that both the canonical and non-canonical pathways might act in concert to regulate the level of inflammation. In addition to direct Wntmediated activation of $\beta$-catenin and other regulatory pathways, TLR2-mediated signals also induce active $\beta$-catenin in DCs and regulate EAE pathology (21). This study showed that TLR-2mediated activation of $\beta$-catenin in DCs promotes IL-10 and retinoic acid (RA) production, which in turn promotes regulatory $\mathrm{T}$ cell responses and suppresses effector $\mathrm{T}$ cell responses. Furthermore, E-cadherin-mediated signaling can also activate $\beta$-catenin in DCs, suppressing chronic inflammation and EAE disease severity $(15,21)$. In addition to DCs, macrophages and microglial cells also play a critical role in initiation and resolution of EAE pathogenesis $(53,54)$. Although these observations indicated the role for Wnts and TLRs in modulating DC activity during EAE, it is quite possible that increased Wnt ligand expression could regulate functions of macrophage and microglial cells and limit EAE pathology. Further studies on cellular source and targets of Wnt ligands during different stages of EAE are necessary to delineate the regulatory role of the $\mathrm{Wnt} / \beta$-catenin pathway during autoimmune neuroinflammation.

Several recent studies have characterized the critical role of Wnt $/ \beta$-catenin signaling in the pathogenesis of chronic inflammatory diseases such as lupus, psoriasis, and rheumatoid arthritis. For example, using murine models for lupus, it has been shown that Dickkopf-1 (DKK1), a negative regulator of the $\mathrm{Wnt} / \beta$ catenin pathway, is highly upregulated in the serum during lupus progression (55). Similarly, serum samples from lupus patients showed increased levels of DKK1, suggesting altered canonical Wnt signaling in the development of lupus (56). DKK1 overexpression has also been observed in humans in other autoimmune disorders. For example, skin and peripheral blood mononuclear cells expressed increased levels of the Wnt signaling inhibitor in psoriasis (57). However, it is not known whether increases in the levels of negative regulators of the Wnt pathway alter functions of DCs or macrophages.

In contrast to negative regulators of the canonical Wntpathway, aberrant expression of Wnts such as Wnt5a and Wnt7B that activate the non-canonical pathway was observed in the joints of rheumatoid arthritis patients (58). Conversely, effects of these Wnts on immune cell function are not known. Recent studies also imply downregulation of canonical Wnt signaling receptors in rheumatoid arthritis, as indicated by silencing of Fzd8 by miRNA-375 in arthritis synovial fibroblasts (59). Activation of Wnt signaling in rheumatoid arthritis has been known to induce proliferation of fibroblast-like synoviocytes and increases in synovial inflammation (58). Also, non-canonical pathways may antagonize the canonical $\beta$-catenin signaling pathway by downregulating $\beta$-catenin protein expression $(60,61)$. Thus, it appears that upregulation of non-canonical Wnt signaling that leads to downregulation of canonical Wnt signaling may be occurring in rheumatoid arthritis to induce high levels of synovial inflammation. Wnt signaling has been known to play a role in cell renewal and keratinocyte differentiation, and the Wnt/Ca ${ }^{2+}$ non-canonical pathway has been correlated with the pathophysiology of psoriasis (62). The overexpression of Wnt5A and Fzd5 in psoriasis may denote the targeting of non-canonical Wnt pathway elements as an efficacious tactic in the remediation of psoriatic symptoms. Thus, activating the canonical Wnt/ $\beta$ catenin pathway and suppressing non-canonical Wnt signaling represent a potential therapeutic approach toward suppressing inflammation and limiting immune-mediated pathology.

\section{Wnt/ $\beta$-CATENIN SIGNALING IN DCs MODULATES ANTITUMOR IMMUNITY}

Although activation of $\beta$-catenin signaling in DCs is critical for mucosal tolerance and resolving chronic inflammatory conditions, tumor cells, and some pathogenic microbes exploit this pathway to effectively suppress DC-mediated host immune responses $(20,21,63)$. Tumors use different mechanisms to evade immunity, including suppressing DC-induced antitumor immune responses in the tumor microenvironment (TME) (64, 65). Numerous studies have shown increased Wnt expression in 
tumors and linked dysregulation of the Wnt pathway with tumor progression (66). However, most studies focused on how the Wnt signaling cascade regulates tumor development, progression, and metastasis (67-70). The role of $\mathrm{Wnt} / \beta$-catenin signaling in immune cells in the TME and its effect on host antitumor immunity and tumor-induced immune tolerance remain unknown. Recent studies from several labs have shown that increased levels of Wnts in the TME can initiate paracrine signaling and modulate host antitumor immunity $(63,71-75)$.

Using different murine tumor models, we have shown that increased Wnt ligands in tumors program DCs to produce RA and IL-10, which promotes immune suppression by inducing regulatory $\mathrm{T}$ cell responses (63). Mechanistically, this is also due to activation of $\beta$-catenin signaling in TME DCs and their ability to up regulate immune-regulatory factors such as IDO, IL-10, and Raldh $(63,71,73,75)$. Furthermore, TME DCs express high levels of IL- 10 and TGF- $\beta$ that promotes immune tolerance through induction of IL-10-producing $\mathrm{CD}^{+}$and $\mathrm{CD}^{+} \mathrm{T}$ cells (63). Interestingly, IL-10 production by TME DCs is dependent on the $\beta$-catenin/TCF4 signaling axis $(63,71,75$, 76 ) and $\mathrm{mTOR} / \beta$-catenin signaling pathway (71). These studies further suggest that Wnt-mediated activation of $\beta$-catenin in TME DCs is critical for inducing tumor tolerance. Accordingly, conditional deletion of $\beta$-catenin or LRP5/6 in DCs markedly reduces regulatory $\mathrm{T}$ cell responses with increased effector CD8 $\mathrm{T}$ cell responses, leading to suppression of tumor growth (63, 75). Furthermore, blocking Wnt interaction with LRP5/6 and Fzd using small molecule inhibitors in tumor-bearing mice showed increased capture of tumor-associated antigen by DCs. This led to increased cross-priming of CD8 T cells and resulted in reduced tumor burden. Interestingly, recent study showed that melanoma-intrinsic $\beta$-catenin signaling suppresses antitumor immunity by regulating DC migration (74).

In addition, the TME also programs DC function by regulating its activation and maturation through diverse mechanisms, including expression of costimulatory (CD80, CD86, and CD40) and co-inhibitory (PD-L1 and PD-L2) molecules (64). Our data show that deletion of $\beta$-catenin in DCs results in increased activation of DCs in the TME with increased surface expression of costimulatory molecules and decreased expression of coinhibitory molecules (63). In addition to inducing regulatory $\mathrm{T}$ cells, $\beta$-catenin activation in DCs can also affect cross-priming of $\mathrm{CD}^{+} \mathrm{T}$ cell responses against tumors $(71,75)$. Though $\beta$-catenin is known to suppress DC activation and cross-priming (77), it remains to be determined whether $\beta$-catenin directly regulates DC activation or does so indirectly via RA and IL-10 (63).

Since Wnt-mediated activation of $\beta$-catenin in the TME promotes tumor progression through multiple mechanisms, pharmacological suppression of the $\beta$-catenin pathway represents a promising target for effective antitumor immunotherapy. Accordingly, treatment of mice with inhibitors that blocks the $\beta$-catenin/TCF pathway results in delayed tumor growth through suppression of regulatory $\mathrm{T}$ cell responses and increased antitumor CD4 and CD8 T cell responses $(63,71-73,75)$. Collectively, these observations suggest that Wnt family of ligands in the TME can initiate tumor-intrinsic $\beta$-catenin signaling and DC-intrinsic $\beta$-catenin signaling, and both contributing to immune suppression through different effector mechanisms. Blocking the Wnt $/ \beta$-catenin pathway could represent a promising therapeutic approach toward breaking tumor-mediated immune suppression and augmenting antitumor immunity.

\section{REGULATION OF Wnt/ $\beta$-CATENIN SIGNALING IN DCs: NOVEL TARGETS FOR IMMUNOTHERAPIES}

The Wnt/ $\beta$-catenin pathway in DCs is a key player in the pathogenesis of a wide amalgam of inflammatory and infectious diseases (10). Wnt $/ \beta$-catenin pathway thus serves as a potential therapeutic target where activation results in the suppression of inflammatory pathologies, and suppression is beneficial in augmenting antitumor immunity (Figure 2) $(21,49,63,71-73$, $75)$. Small molecules that modulate the $\mathrm{Wnt} / \beta$-catenin pathway have been extensively researched for use against various pathological conditions $(70,78)$ (Figure 3). In this regard, a recent study of ours has shown that pharmacological activation of canonical Wnt/ $\beta$-catenin signaling is beneficial in reducing EAE and associated CNS pathology (49). Mechanistic investigations about various pathways related to Wnt signaling have provided additional therapeutic targets for activation or suppression of canonical Wnt signaling in diseases (Figure 3). In addition to direct inhibition or activation of the canonical Wnt pathway, suppression of non-canonical Notch signaling, which can antagonize canonical Wnt signaling, may be beneficial in regressing symptoms of autoimmune disease. Non-canonical Wnt ligands Wnt5a and Wnt7b were upregulated in the joints of rheumatoid arthritis patients, and the non-canonical Wnt $/ \mathrm{Ca}^{2+}$ pathway has been associated with the pathophysiology of psoriasis $(58,62)$. Pharmacological inhibition of Wnt5A, for example, may remediate inflammation and other symptoms in arthritis or psoriasis patients.

Optimal functioning of $\mathrm{Wnt} / \beta$-catenin pathway in different type of non-immune and immune cells including DCs is vital for a range of cellular functions and homeostasis. Consequently, targeting Wnt/ $\beta$-catenin pathway specifically in DCs and particularly in those DCs, which play a central role in the induction of autoimmune pathology or tumor progression is critical for successful therapeutic intervention to minimize off-target side effects. Therefore, it is crucial to understand the biology of different subsets of DCs and how Wnt/ $\beta$-catenin pathway in these DCs subsets contributes to normal health and immunopathology. Moreover, further studies on understanding the biology Wnt $/ \beta$ catenin signaling in other immune cells such as $\mathrm{B}$ cells, $\mathrm{CD} 8^{+} \mathrm{T}$ cells and different subsets of $\mathrm{CD} 4^{+} \mathrm{T}$ cells are warranted for better therapeutic targeting of this pathway in autoimmunity and cancer. In this regard, recent advances in targeted delivery of small molecules and biologics represent a novel therapeutic approach to deliver Wnt/ $\beta$-catenin signaling modulator specifically to DCs for clinical management of autoimmunity and cancer. Additionally, combining DCs-specific Wnt/ $\beta$-catenin pathway inhibition with recent successful therapies targeting immune checkpoints represents a promising approach to promote antitumor immune responses. 


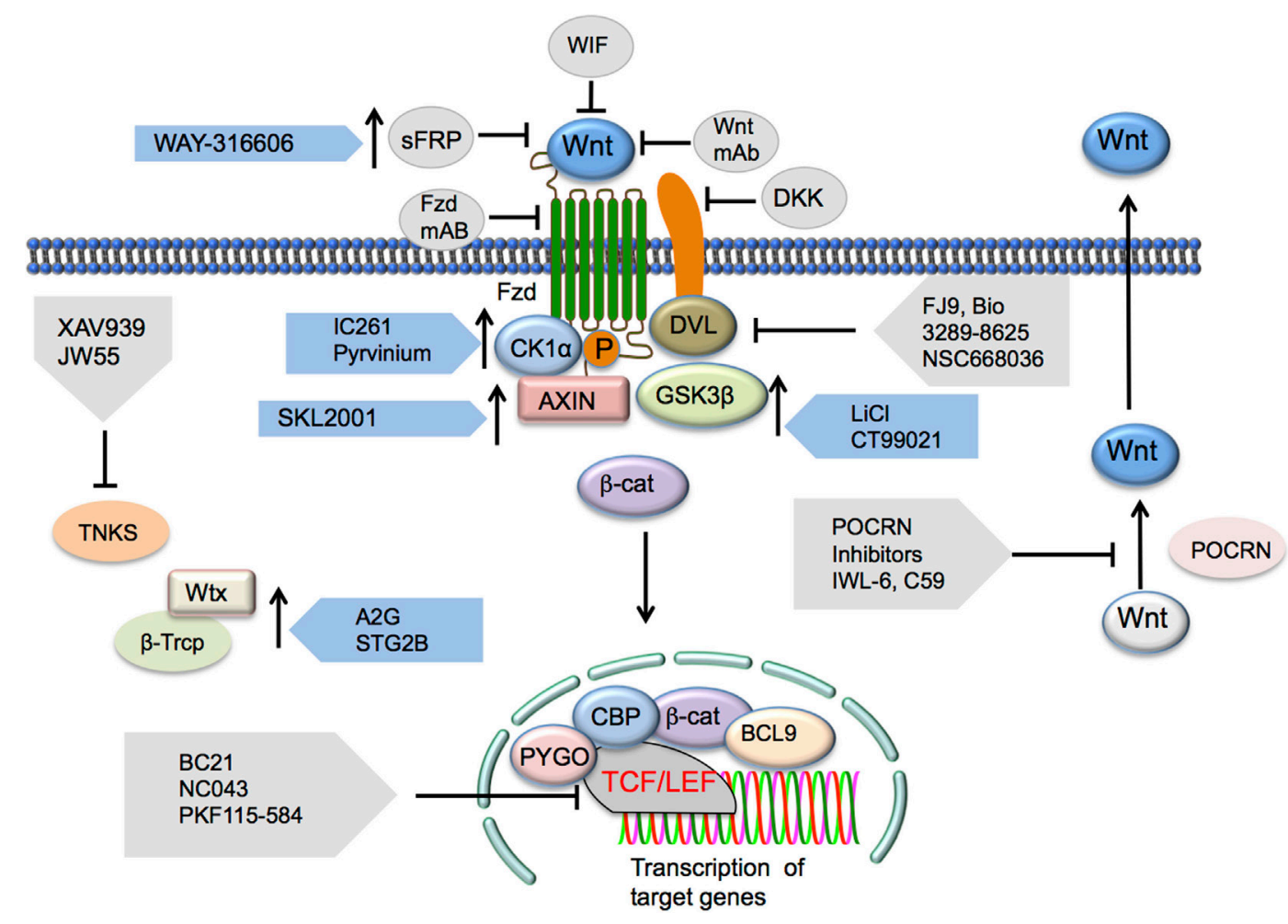

FIGURE 3 | Potential Wnt/ $\boldsymbol{\beta}$-catenin signaling therapeutic targets. Inhibition of the canonical Wnt/ $\beta$-catenin pathway may occur via targeting a number of the pathway's elements. Wnt ligand antagonists include SFRP, WIF, or mAbs generated against the ligands. Frizzled receptor interaction inhibition may occur through the administration of Fzd mAbs as well. DKK1 has been shown to elicit inhibition of LRP co-receptor function. Disheveled (DVL) interactions with Wnt signaling receptors may also be inhibited via FJ9, Bio 3289-8625, or NSC668036. Downstream inhibition may occur through inhibition of $\beta$-catenin and TCF/LEF interactions by a number of different inhibitors. Other Wnt signaling-activating components that may be inhibited include TNKS, an inhibitor of axin stability, and PORCN, a stimulator of Wnt secretion. Inhibition of this pathway may also be the result of the presence of activators of elements that regulate the functions of this pathway that induce the upregulation or increase in function of these regulatory elements. Such activators include those targeting the Ser/Thr kinases CK1 $\alpha$ (IC261 and pyrvinium) and GSK3 $\beta$ (lithium, CT99021, and BIO), the axin scaffolding protein (SKL2000), or $\beta$-Trcp ( $\Delta 2 \mathrm{G}$, STG28). Abbreviations: SFRP, secreted frizzled-related protein-1; WIF, Wnt inhibitory protein; Fzd, Frizzled; mAbs, monoclonal antibodies; DKK1, Dickkopf-related protein 1; LRP, low-density lipoprotein receptor-related protein; TCF/LEF, T cell factor/lymphoid enhancer factor; TNKS, tankyrase 1 ; PORCN, porcupine; CK1 $\alpha$, casein kinase $1 \alpha$; GSK3 $\beta$, glycogen synthase kinase 3 $\beta$; $\beta$-Trcp, $\beta$-transducin repeat-containing protein.

\section{SUMMARY}

The normal immune system functions by instigating an effective immune response against infectious agents while promoting tolerance toward commensal microbiota and self-antigens. The Wnt/ $\beta$-catenin pathway participates in this balance through diverse mechanisms including the generation of tolerogenic DCs to regulate inflammatory responses. Inadequacies in the signaling of the $\mathrm{Wnt} / \beta$-catenin pathway may result in allergic and autoimmune diseases through chronic and uncontrolled activation of the immune system or cancer due to inadequate immune surveillance resulting from excessive immune tolerance. Thus, $\mathrm{Wnt} / \beta$-catenin signaling serves as a molecular switch between opposing immune functions, and targeting different elements of this pathway may provide therapeutic benefits in the remediation of autoimmune diseases, cancer, and infectious diseases. While it is clear that Wnt-signaling programs DCs to induce robust regulatory $\mathrm{T}$ cell responses and cytokines to contain inflammation, several important questions remain unanswered. It is unknown, for example, how the canonical and non-canonical pathways act in tandem to regulate immunity and tolerance, and how much crosstalk is involved with other pathways involved in the regulation of immune responses. Dissecting many of these issues around $\mathrm{Wnt} / \beta$-catenin signaling in immune cells potentially helps in developing novel therapeutic strategies against various diseases stemming from an imbalance in the immune system.

\section{AUTHOR CONTRIBUTIONS}

AS and RT have performed bibliographic researches and drafted the manuscript. DS has performed bibliographic researches. SM has performed bibliographic researches and drafted the manuscript.

\section{FUNDING}

We gratefully acknowledge the generous support of the National Institutes of Health (DK097271) in our work. 


\section{REFERENCES}

1. Miller JF, Morahan G. Peripheral T cell tolerance. Annu Rev Immunol (1992) 10:51-69. doi:10.1146/annurev.iy.10.040192.000411

2. Manicassamy S, Pulendran B. Dendritic cell control of tolerogenic responses. Immunol Rev (2011) 241:206-27. doi:10.1111/j.1600-065X.2011.01015.X

3. Medzhitov R. Recognition of microorganisms and activation of the immune response. Nature (2007) 449:819-26. doi:10.1038/nature06246

4. Pulendran B, Tang H, Manicassamy S. Programming dendritic cells to induce $\mathrm{T}(\mathrm{H}) 2$ and tolerogenic responses. Nat Immunol (2010) 11:647-55. doi:10.1038/ni.1894

5. Steinman RM, Nussenzweig MC. Avoiding horror autotoxicus: the importance of dendritic cells in peripheral T cell tolerance. Proc Natl Acad Sci U S A (2002) 99:351-8. doi:10.1073/pnas.231606698

6. Steinman RM, Hawiger D, Nussenzweig MC. Tolerogenic dendritic cells. Annu Rev Immunol (2003) 21:685-711. doi:10.1146/annurev. immunol.21.120601.141040

7. Mosmann TR, Cherwinski H, Bond MW, Giedlin MA, Coffman RL. Two types of murine helper T cell clone. I. Definition according to profiles of lymphokine activities and secreted proteins. J Immunol (1986) 136:2348-57.

8. Murphy CA, Langrish CL, Chen Y, Blumenschein C, Mcclanahan T, Kastelein RA, et al. Divergent pro- and antiinflammatory roles for IL-23 and IL-12 in joint autoimmune inflammation. J Exp Med (2003) 198:1951-7. doi:10.1084/ jem.20030896

9. van Amerongen R, Nusse R. Towards an integrated view of Wnt signaling in development. Development (2009) 136:3205-14. doi:10.1242/dev.033910

10. Clevers H, Nusse R. Wnt/beta-catenin signaling and disease. Cell (2012) 149:1192-205. doi:10.1016/j.cell.2012.05.012

11. Staal FJ, Sen JM. The canonical Wnt signaling pathway plays an important role in lymphopoiesis and hematopoiesis. Eur J Immunol (2008) 38:1788-94. doi:10.1002/eji.200738118

12. MacDonald BT, Tamai $\mathrm{K}, \mathrm{He} \mathrm{X}$. Wnt/beta-catenin signaling: components, mechanisms, and diseases. Dev Cell (2009) 17:9-26. doi:10.1016/j. devcel.2009.06.016

13. Gordon MD, Nusse R. Wnt signaling: multiple pathways, multiple receptors, and multiple transcription factors. J Biol Chem (2006) 281:22429-33. doi:10.1074/jbc.R600015200

14. Kennell J, Cadigan KM. APC and beta-catenin degradation. Adv Exp Med Biol (2009) 656:1-12. doi:10.1007/978-1-4419-1145-2_1

15. Jiang A, Bloom O, Ono S, Cui W, Unternaehrer J, Jiang S, et al. Disruption of E-cadherin-mediated adhesion induces a functionally distinct pathway of dendritic cell maturation. Immunity (2007) 27:610-24. doi:10.1016/j. immuni.2007.08.015

16. Gottardi CJ, Wong E, Gumbiner BM. E-cadherin suppresses cellular transformation by inhibiting beta-catenin signaling in an adhesion-independent manner. J Cell Biol (2001) 153:1049-60. doi:10.1083/jcb.153.5.1049

17. Maher MT, Flozak AS, Stocker AM, Chenn A, Gottardi CJ. Activity of the beta-catenin phosphodestruction complex at cell-cell contacts is enhanced by cadherin-based adhesion. J Cell Biol (2009) 186:219-28. doi:10.1083/ jcb.200811108

18. Torres VA, Tapia JC, Rodriguez DA, Lladser A, Arredondo C, Leyton L, et al. E-cadherin is required for caveolin-1-mediated down-regulation of the inhibitor of apoptosis protein survivin via reduced beta-catenin-Tcf/Lefdependent transcription. Mol Cell Biol (2007) 27:7703-17. doi:10.1128/ MCB.01991-06

19. Casagolda D, Del Valle-Perez B, Valls G, Lugilde E, Vinyoles M, Casado-Vela J, et al. A p120-catenin-CK1 epsilon complex regulates Wnt signaling. J Cell Sci (2010) 123:2621-31. doi:10.1242/jcs.067512

20. Silva-Garcia O, Valdez-Alarcon JJ, Baizabal-Aguirre VM. The Wnt/beta-catenin signaling pathway controls the inflammatory response in infections caused by pathogenic bacteria. Mediators Inflamm (2014) 2014:310183. doi: $10.1155 / 2014 / 310183$

21. Manoharan I, Hong Y, Suryawanshi A, Angus-Hill ML, Sun Z, Mellor AL, et al. TLR2-dependent activation of beta-catenin pathway in dendritic cells induces regulatory responses and attenuates autoimmune inflammation. J Immunol (2014) 193:4203-13. doi:10.4049/jimmunol.1400614

22. Manicassamy S, Ravindran R, Deng J, Oluoch H, Denning TL, Kasturi SP, et al. Toll-like receptor 2-dependent induction of vitamin A-metabolizing enzymes in dendritic cells promotes $\mathrm{T}$ regulatory responses and inhibits autoimmunity. Nat Med (2009) 15:401-9. doi:10.1038/nm.1925

23. Dillon S, Agrawal A, Van Dyke T, Landreth G, Mccauley L, Koh A, et al. A toll-like receptor 2 ligand stimulates Th2 responses in vivo, via induction of extracellular signal-regulated kinase mitogen-activated protein kinase and c-Fos in dendritic cells. J Immunol (2004) 172:4733-43. doi:10.4049/ jimmunol.172.8.4733

24. Dillon S, Agrawal S, Banerjee K, Letterio J, Denning TL, Oswald-Richter K, et al. Yeast zymosan, a stimulus for TLR2 and dectin-1, induces regulatory antigen-presenting cells and immunological tolerance. JClin Invest (2006) 116:916-28. doi:10.1172/JCI27203

25. Yang PY, An HZ, Liu XG, Wen MY, Zheng YY, Rui YC, et al. The cytosolic nucleic acid sensor LRRFIP1 mediates the production of type I interferon via a beta-catenin-dependent pathway. Nat Immunol (2010) 11:487-U450. doi:10.1038/ni.1876

26. Hay N. Interplay between FOXO, TOR, and Akt. Biochim Biophys Acta (2011) 1813:1965-70. doi:10.1016/j.bbamcr.2011.03.013

27. Gantner BN, Jin HL, Qian F, Hay N, He B, Ye RD. The Akt1 isoform is required for optimal IFN-beta transcription through direct phosphorylation of beta-catenin. J Immunol (2012) 189:3104-11. doi:10.4049/ jimmunol.1201669

28. Sun J, Hobert ME, Duan YL, Rao AS, He TC, Chang EB, et al. Crosstalk between NF-kappa B and beta-catenin pathways in bacterial-colonized intestinal epithelial cells. Am J Physiol Gastrointest Liver Physiol (2005) 289:G129-37. doi:10.1152/ajpgi.00515.2004

29. Qian C, Qian L, Yu YZ, An HZ, Guo ZH, Han YM, et al. Fas signal promotes the immunosuppressive function of regulatory dendritic cells via the ERK/ beta-catenin pathway. J Biol Chem (2013) 288:27825-35. doi:10.1074/jbc. M112.425751

30. Lugt BV, Beck ZT, Fuhlbrigge RC, Hacohen N, Campbell JJ, Boes M. TGFbeta suppresses beta-catenin-dependent tolerogenic activation program in dendritic cells. PLoS One (2011) 6(5):e20099. doi:10.1371/journal. pone.0020099

31. Capietto AH, Kim S, Sanford DE, Linehan DC, Hikida M, Kumosaki T, et al. Down-regulation of PLC gamma 2-beta-catenin pathway promotes activation and expansion of myeloid-derived suppressor cells in cancer. J Exp Med (2013) 210:2257-71. doi:10.1084/jem.20130281

32. Korinek V, Barker N, Moerer P, Van Donselaar E, Huls G, Peters PJ, et al. Depletion of epithelial stem-cell compartments in the small intestine of mice lacking Tcf-4. Nat Genet (1998) 19:379-83. doi:10.1038/1270

33. Hoffman J, Kuhnert F, Davis CR, Kuo CJ. Wnts as essential growth factors for the adult small intestine and colon. Cell Cycle (2004) 3:554-7. doi:10.4161/ cc.3.5.858

34. Ireland H, Kemp R, Houghton C, Howard L, Clarke AR, Sansom OJ, et al. Inducible Cre-mediated control of gene expression in the murine gastrointestinal tract: effect of loss of beta-catenin. Gastroenterology (2004) 126:1236-46. doi:10.1053/j.gastro.2004.03.020

35. Kuhnert F, Davis CR, Wang HT, Chu P, Lee M, Yuan J, et al. Essential requirement for Wnt signaling in proliferation of adult small intestine and colon revealed by adenoviral expression of Dickkopf-1. Proc Natl Acad Sci U S A (2004) 101:266-71. doi:10.1073/pnas.2536800100

36. Fevr T, Robine S, Louvard D, Huelsken J. Wnt/beta-catenin is essential for intestinal homeostasis and maintenance of intestinal stem cells. Mol Cell Biol (2007) 27:7551-9. doi:10.1128/MCB.01034-07

37. Korinek V, Barker N, Morin PJ, Vanwichen D, Deweger R, Kinzler KW, et al. Constitutive transcriptional activation by a beta-catenin-Tcf complex in APC(-/-) colon carcinoma. Science (1997) 275:1784-7. doi:10.1126/ science.275.5307.1784

38. Morin PJ, Sparks AB, Korinek V, Barker N, Clevers H, Vogelstein B, et al. Activation of beta-catenin-Tcf signaling in colon cancer by mutations in beta-catenin or APC. Science (1997) 275:1787-90. doi:10.1126/ science. 275.5307 .1787

39. Kolligs FT, Bommer G, Goke B. Wnt/beta-catenin/Tcf signaling: a critical pathway in gastrointestinal tumorigenesis. Digestion (2002) 66:131-44. doi:10.1159/000066755

40. Gregorieff A, Pinto D, Begthel H, Destree O, Kielman M, Clevers H. Expression pattern of Wnt signaling components in the adult intestine. Gastroenterology (2005) 129:626-38. doi:10.1053/j.gastro.2005.06.007 
41. Manicassamy S, Reizis B, Ravindran R, Nakaya H, Salazar-Gonzalez RM, Wang YC, et al. Activation of beta-catenin in dendritic cells regulates immunity versus tolerance in the intestine. Science (2010) 329:849-53. doi:10.1126/ science. 1188510

42. Shan MM, Gentile M, Yeiser JR, Walland AC, Bornstein VU, Chen K, et al. Mucus enhances gut homeostasis and oral tolerance by delivering immunoregulatory signals. Science (2013) 342:447-53. doi:10.1126/ science. 1237910

43. Rescigno M, Di Sabatino A. Dendritic cells in intestinal homeostasis and disease. J Clin Invest (2009) 119:2441-50. doi:10.1172/JCI39134

44. Ganguly D, Haak S, Sisirak V, Reizis B. The role of dendritic cells in autoimmunity. Nat Rev Immunol (2013) 13:566-77. doi:10.1038/nri3477

45. Terry RL, Ifergan I, Miller SD. Experimental autoimmune encephalomyelitis in mice. Methods Mol Biol (2016) 1304:145-60. doi:10.1007/7651_2014_88

46. Bettelli E, Oukka M, Kuchroo VK. T(H)-17 cells in the circle of immunity and autoimmunity. Nat Immunol (2007) 8:345-50. doi:10.1038/ni0407-345

47. Huseby ES, Liggitt D, Brabb T, Schnabel B, Ohlen C, Goverman J. A pathogenic role for myelin-specific $\mathrm{CD} 8(+) \mathrm{T}$ cells in a model for multiple sclerosis. J Exp Med (2001) 194:669-76. doi:10.1084/jem.194.5.669

48. Ji QY, Goverman J. Experimental autoimmune encephalomyelitis mediated by CD8(+) T cells. How Do We Best Employ Animal Models for Type 1 Diabetes and Multiple Sclerosis? (Vol. 1103) (2007). p. 157-66.

49. Suryawanshi A, Manoharan I, Hong Y, Swafford D, Majumdar T, Taketo MM, et al. Canonical wnt signaling in dendritic cells regulates th $1 /$ th 17 responses and suppresses autoimmune neuroinflammation. J Immunol (2015) 194:3295-304. doi:10.4049/jimmunol.1402691

50. Marchetti B, Pluchino S. Wnt your brain be inflamed? Yes, it Wnt! Trends Mol Med (2013) 19:144-56. doi:10.1016/j.molmed.2012.12.001

51. Valencia J, Hernandez-Lopez C, Martinez VG, Hidalgo L, Zapata AG, Vicente A, et al. Wnt5a skews dendritic cell differentiation to an unconventional phenotype with tolerogenic features. J Immunol (2011) 187:4129-39. doi:10.4049/ jimmunol.1101243

52. Oderup C, Lajevic M, Butcher EC. Canonical and noncanonical Wnt proteins program dendritic cell responses for tolerance. J Immunol (2013) 190:6126-34. doi:10.4049/jimmunol.1203002

53. Heneka MT. Macrophages derived from infiltrating monocytes mediate autoimmune myelin destruction. JExp Med (2014) 211:1500. doi:10.1084/ jem.2118insight 1

54. Yamasaki R, Lu H, Butovsky O, Ohno N, Rietsch AM, Cialic R, et al. Differential roles of microglia and monocytes in the inflamed central nervous system. J Exp Med (2014) 211:1533-49. doi:10.1084/jem.20132477

55. Tveita AA, Rekvig OP. Alterations in Wnt pathway activity in mouse serum and kidneys during lupus development. Arthritis Rheum (2011) 63:513-22. doi:10.1002/art.30116

56. Wang XD, Huang XF, Yan QR, Bao CD. Aberrant activation of the WNT/ beta-catenin signaling pathway in lupus nephritis. PLoS One (2014) 9:e84852. doi:10.1371/journal.pone.0084852

57. Seifert O, Soderman J, Skarstedt M, Dienus O, Matussek A. Increased expression of the Wnt signalling inhibitor Dkk-1 in non-lesional skin and peripheral blood mononuclear cells of patients with plaque psoriasis. Acta Derm Venereol (2015) 95:407-10. doi:10.2340/00015555-1970

58. Miao CG, Yang YY, He X, Li XF, Huang C, Huang Y, et al. Wnt signaling pathway in rheumatoid arthritis, with special emphasis on the different roles in synovial inflammation and bone remodeling. Cell Signal (2013) 25:2069-78. doi:10.1016/j.cellsig.2013.04.002

59. Miao CG, Shi WJ, Xiong YY, Yu H, Zhang XL, Qin MS, et al. miR-375 regulates the canonical Wnt pathway through FZD8 silencing in arthritis synovial fibroblasts. Immunol Lett (2015) 164:1-10. doi:10.1016/j.imlet.2015. 01.003

60. Liang H, Chen Q, Coles AH, Anderson SJ, Pihan G, Bradley A, et al. Wnt5a inhibits B cell proliferation and functions as a tumor suppressor in hematopoietic tissue. Cancer Cell (2003) 4:349-60. doi:10.1016/S1535-6108(03) 00268-X

61. Liang H, Coles AH, Zhu Z, Zayas J, Jurecic R, Kang J, et al. Noncanonical Wnt signaling promotes apoptosis in thymocyte development. J Exp Med (2007) 204:3077-84. doi:10.1084/jem.20062692
62. Kelotra A, Gokhale SM, Kelotra S, Mukadam V, Nagwanshi K, Bandaru S, et al. Alkyloxy carbonyl modified hexapeptides as a high affinity compounds for Wnt5A protein in the treatment of psoriasis. Bioinformation (2014) 10:743-9. doi:10.6026/97320630010743

63. Hong Y, Manoharan I, Suryawanshi A, Majumdar T, Angus-Hill ML, Koni PA, et al. beta-catenin promotes regulatory $\mathrm{T}$-cell responses in tumors by inducing vitamin A metabolism in dendritic cells. Cancer Res (2015) 75:656-65. doi:10.1158/0008-5472.CAN-14-2377

64. Rabinovich GA, Gabrilovich D, Sotomayor EM. Immunosuppressive strategies that are mediated by tumor cells. Annu Rev Immunol (2007) 25:267-96. doi:10.1146/annurev.immunol.25.022106.141609

65. Palucka K, Banchereau J. Cancer immunotherapy via dendritic cells. Nat Rev Cancer (2012) 12:265-77. doi:10.1038/nrc3258

66. Reya T, Clevers H. Wnt signalling in stem cells and cancer. Nature (2005) 434:843-50. doi:10.1038/nature03319

67. Larue L, Delmas V. The WNT/Beta-catenin pathway in melanoma. Front Biosci (2006) 11:733-42. doi:10.2741/1831

68. Fodde R, Brabletz T. Wnt/beta-catenin signaling in cancer stemness and malignant behavior. Curr Opin Cell Biol (2007) 19:150-8. doi:10.1016/j. ceb.2007.02.007

69. Macheda ML, Stacker SA. Importance of Wnt signaling in the tumor stroma microenvironment. Curr Cancer Drug Targets (2008) 8:454-65. doi:10.2174/156800908785699324

70. Anastas JN, Moon RT. WNT signalling pathways as therapeutic targets in cancer. Nat Rev Cancer (2013) 13:11-26. doi:10.1038/nrc3419

71. Liang X, Fu C, Cui W, Ober-Blobaum JL, Zahner SP, Shrikant PA, et al. beta-catenin mediates tumor-induced immunosuppression by inhibiting cross-priming of CD8(+) T cells. J Leukoc Biol (2014) 95:179-90. doi:10.1189/ jlb.0613330

72. Fu C, Liang X, Cui W, Ober-Blobaum JL, Vazzana J, Shrikant PA, et al. beta-Catenin in dendritic cells exerts opposite functions in cross-priming and maintenance of CD8+ T cells through regulation of IL-10. Proc Natl Acad Sci U S A (2015) 112:2823-8. doi:10.1073/pnas.1414167112

73. Holtzhausen A, Zhao F, Evans KS, Tsutsui M, Orabona C, Tyler DS, et al. Melanoma-derived Wnt5a promotes local dendritic-cell expression of IDO and immunotolerance: opportunities for pharmacologic enhancement of immunotherapy. Cancer Immunol Res (2015) 3:1082-95. doi:10.1158/23266066.CIR-14-0167

74. Spranger S, Bao R, Gajewski TF. Melanoma-intrinsic beta-catenin signalling prevents anti-tumour immunity. Nature (2015) 523:231-5. doi:10.1038/ nature14404

75. Hong Y, Manoharan I, Suryawanshi A, Shanmugam A, Swafford D, Ahmad S, et al. Deletion of LRP5 and LRP6 in dendritic cells enhances antitumor immunity. Oncoimmunology (2016) 5:e1115941. doi:10.1080/21624 02X.2015.1115941

76. Suryawanshi A, Manicassamy S. Tumors induce immune tolerance through activation of beta-catenin/TCF4 signaling in dendritic cells: a novel therapeutic target for cancer immunotherapy. Oncoimmunology (2015) 4:e1052932. doi:10.1080/2162402X.2015.1052932

77. Manicassamy S, Pulendran B. Retinoic acid-dependent regulation of immune responses by dendritic cells and macrophages. Semin Immunol (2009) 21:22-7. doi:10.1016/j.smim.2008.07.007

78. Chen B, Dodge ME, Tang W, Lu J, Ma Z, Fan CW, et al. Small molecule-mediated disruption of Wnt-dependent signaling in tissue regeneration and cancer. Nat Chem Biol (2009) 5:100-7. doi:10.1038/nchembio.137

Conflict of Interest Statement: The authors declare that the research was conducted in the absence of any commercial or financial relationships that could be construed as a potential conflict of interest.

Copyright $\odot 2016$ Suryawanshi, Tadagavadi, Swafford and Manicassamy. This is an open-access article distributed under the terms of the Creative Commons Attribution License (CC BY). The use, distribution or reproduction in other forums is permitted, provided the original author(s) or licensor are credited and that the original publication in this journal is cited, in accordance with accepted academic practice. No use, distribution or reproduction is permitted which does not comply with these terms. 